\title{
DOUBLY WARPED PRODUCT SUBMANIFOLDS OF A RIEMANNIAN MANIFOLD OF QUASI-CONSTANT CURVATURE
}

\author{
BY
}

SIBEL SULAR

\begin{abstract}
We establish a sharp inequality for a doubly warped product submanifold in a Riemannian manifold of quasi-constant curvature.

Mathematics Subject Classification 2010: 53C40, 53B25.

Key words: doubly warped product, Riemannian manifold of quasi-constant curva-
\end{abstract} ture.

\section{Introduction}

The notion of a Riemannian manifold $(M, g)$ of quasi-constant curvature was introduced by CHEN and YANO [6] and it defines a Riemannian manifold whose curvature tensor $R$ satisfies the condition

$$
\begin{aligned}
R(X, Y, Z, W)= & a[g(Y, Z) g(X, W)-g(X, Z) g(Y, W)] \\
& +b[g(X, W) T(Y) T(Z)-g(X, Z) T(Y) T(W) \\
& +g(Y, Z) T(X) T(W)-g(Y, W) T(X) T(Z)],
\end{aligned}
$$

where $a$ and $b$ are scalar functions and $T$ is a 1-form given by $g(X, P)=$ $T(X)$, with $P$ a fixed unit vector field. It is easy to see that if $R$ is of the form (1), then the manifold is conformally flat. If $b=0$, then the manifold is called to be a space of constant curvature.

An $n$-dimensional $(n>2)$ non-flat Riemannian manifold $M$ is said to be a quasi-Einstein manifold (see [1]) if its Ricci tensor $S$ satisfies the condition $S(X, Y)=\alpha g(X, Y)+\beta A(X) A(Y)$, where $\alpha$ and $\beta$ are scalar functions such 
that $\beta \neq 0$ and $A$ is a non-zero 1-form denoted by $g(X, U)=A(X)$, for any vector field $X$ and where $U$ is a fixed unit vector field. It is easy to see that any Riemannian manifold of quasi-constant curvature is a quasi-Einstein manifold.

Let $\left(M_{1}, g_{1}\right)$ and $\left(M_{2}, g_{2}\right)$ be two Riemannian manifolds and $f_{1}, f_{2}$ be positive, differentiable functions on $M_{1}$ and $M_{2}$, respectively. The doubly warped product $M={ }_{f_{2}} M_{1} \times{ }_{f_{1}} M_{2}$ (see [10]) is the product manifold $M_{1} \times M_{2}$ equipped with the metric $g=f_{2}^{2} g_{1}+f_{1}^{2} g_{2}$. More precisely, if $\pi_{1}: M_{1} \times M_{2} \rightarrow$ $M_{1}$ and $\pi_{2}: M_{1} \times M_{2} \rightarrow M_{2}$ are canonical projections, then the metric $g$ is defined by $g=\left(f_{2} \circ \pi_{2}\right)^{2} \pi_{1}^{*} g_{1}+\left(f_{1} \circ \pi_{1}\right)^{2} \pi_{2}^{*} g_{2}$. The functions $f_{1}$ and $f_{2}$ are called warping functions. If either $f_{1} \equiv 1$ or $f_{2} \equiv 1$, but not both, then we get a warped product. If both $f_{1} \equiv 1$ and $f_{2} \equiv 1$, then we obtain a Riemannian product manifold. If neither $f_{1}$ nor $f_{2}$ is constant, then we have a non-trivial doubly warped product (see [10]).

For a doubly warped product $f_{2} M_{1} \times_{f_{1}} M_{2}$, let $D_{1}$ and $D_{2}$ denote the distributions obtained from the vectors tangent to leaves and fibres, respectively.

Assume that $x: f_{2} M_{1} \times f_{1} M_{2} \rightarrow N$ is an isometric immersion of a doubly warped product $f_{2} M_{1} \times f_{1} M_{2}$ into a Riemannian manifold $N$. We denote by $\sigma$ the second fundamental form of $x$ and by $H_{i}=\frac{1}{n_{i}}$ trace $_{i}$ the partial mean curvatures, where $\operatorname{trace}_{i}$ is the trace of $\sigma$ restricted to $M_{i}$ and $n_{i}=\operatorname{dim} M_{i}$ $(i=1,2)$. The immersion $x$ is called mixed totally geodesic if $\sigma(X, Z)=0$, for any vector fields $X$ and $Z$ tangent to $D_{1}$ and $D_{2}$, respectively.

In [5], CHEn proved the following result for a warped product submanifold of a Riemannian manifold of constant sectional curvature:

Theorem 1.1. Let $x: M_{1} \times_{f} M_{2} \rightarrow N(c)$ be an isometric immersion of an $n$-dimensional warped product $M_{1} \times_{f} M_{2}$ into an m-dimensional Riemannian manifold $N(c)$ of constant sectional curvature $c$. Then we have

$$
\frac{\Delta f}{f} \leq \frac{n^{2}}{4 n_{2}}\|H\|^{2}+n_{1} c
$$

where $n_{i}=\operatorname{dim} M_{i}, n=n_{1}+n_{2}, \Delta$ is the Laplacian operator of $M_{1}$. The equality case of (2) holds identically if and only if $x$ is a mixed totally geodesic immersion and $n_{1} H_{1}=n_{2} H_{2}$, where $H_{i}, i=1,2$ are the partial mean curvature vectors.

As a generalization of Chen's result, in [8], ÖzGür and Murathan considered warped product submanifolds of a Riemannian manifold of quasi- 
constant curvature and obtained the following sharp inequality for a warped product isometrically immersed in a Riemannian manifold of quasi-constant curvature:

Theorem 1.2. Let $x: M_{1} \times_{f} M_{2} \rightarrow N^{m}$ be an isometric immersion of an $n$-dimensional warped product $M_{1} \times{ }_{f} M_{2}$ into an m-dimensional Riemannian manifold $N^{m}$ of quasi-constant curvature. Then we have:

$$
\begin{aligned}
\frac{\Delta f}{f} \leq & \frac{n^{2}}{4 n_{2}}\|H\|^{2}+n_{1} a-\frac{b}{n_{2}} \sum_{1 \leq i \leq n_{1}} \sum_{n_{1}+1 \leq s \leq n}\left(T\left(e_{i}\right)^{2}+T\left(e_{s}\right)^{2}\right) \\
& +\frac{b}{n_{2}}(n-1)\left\|P^{\top}\right\|^{2},
\end{aligned}
$$

where $n_{i}=\operatorname{dim} M_{i}$. The equality sign of (3) holds identically if and only if the immersion $x$ is mixed totally geodesic with $\operatorname{tr} \sigma_{1}=\operatorname{tr} \sigma_{2}$.

Recently, in [7], Olteanu established the following general inequality for arbitrary isometric immersions of doubly warped product manifolds in arbitrary Riemannian manifolds:

Theorem 1.3. Let $x$ be an isometric immersion of an $n$-dimensional doubly warped product $M={ }_{f_{2}} M_{1} \times_{f_{1}} M_{2}$ into an $m$-dimensional arbitrary Riemannian manifold $\widetilde{M}$. Then we have

$$
n_{2} \frac{\Delta_{1} f_{1}}{f_{1}}+n_{1} \frac{\Delta_{2} f_{2}}{f_{2}} \leq \frac{n^{2}}{4}\|H\|^{2}+n_{1} n_{2} \max \widetilde{K},
$$

where $n_{i}=\operatorname{dim} M_{i}, n=n_{1}+n_{2}, \Delta_{i}$ is the Laplacian operator of $M_{i}, i=1,2$ and $\max \widetilde{K}(p)$ denotes the maximum of the sectional curvature function of $\widetilde{M}$ restricted to 2-plane sections of the tangent space $T_{p} M$ of $M$ at each point $p$ in $M$. Moreover, the equality case of (4) holds if and only if the following two statements hold:

(1) $x$ is a mixed totally geodesic immersion satisfying $n_{1} H_{1}=n_{2} H_{2}$, where $H_{i}, i=1,2$ are the partial mean curvature vectors of $M_{i}$.

(2) at each point $p=\left(p_{1}, p_{2}\right) \in M$, the sectional curvature function $\widetilde{K}$ of $\widetilde{M}$ satisfies $\widetilde{K}(u, v)=\max \widetilde{K}(p)$ for each unit vector $u \in T_{p_{1}} M_{1}$ and each unit vector $v \in T_{p_{2}} M_{2}$.

Moreover, in [9], the present author and ÖzGüR studied $C$-totally real doubly warped product submanifolds in $(\kappa, \mu)$-contact space forms and nonSasakian $(\kappa, \mu)$-contact metric manifolds. 
Motivated by the studies of the above mentioned authors, in the present paper, we establish a sharp inequality for a doubly warped product submanifold in a Riemannian manifold of quasi-constant curvature.

The paper is organized as follows: Section 2 is devoted to preliminaries. In section 3 , we give a sharp inequality for a doubly warped product submanifold in a Riemannian manifold of quasi-constant curvature.

\section{Preliminaries}

Let $M$ be an $n$-dimensional Riemannian manifold and $p \in M$. Denote by $K(\pi)$ or $K(u, v)$ the sectional curvature of $M$ associated with a plane section $\pi \subset T_{p} M$, where $\{u, v\}$ is an orthonormal basis of $\pi$. For any $n$ dimensional subspace $L \subseteq T_{p} M, 2 \leq n \leq m$, its scalar curvature $\tau(L)$ is denoted by $\tau(L)=\sum_{1 \leq i<j \leq n} K\left(e_{i} \wedge e_{j}\right)$, where $\left\{e_{1}, \ldots, e_{n}\right\}$ is any orthonormal basis of $L$ (see [4]). When $L=T_{p} M$, then the scalar curvature $\tau(L)$ is just the scalar curvature $\tau(p)$ of $M$ at $p$.

For an $n$-dimensional submanifold $M$ in a Riemannian $m$-manifold $N$, we denote by $\nabla$ and $\widetilde{\nabla}$ the Levi-Civita connections of $M$ and $N$, respectively. The Gauss and Weingarten formulas are given by $\widetilde{\nabla}_{X} Y=\nabla_{X} Y+\sigma(X, Y)$ and $\widetilde{\nabla}_{X} \xi=-A_{\xi} X+\nabla_{X}^{\perp} Y$, respectively, for vector fields $X, Y$ tangent to $M$ and $\xi$ normal to $M$, where $\sigma$ denotes the second fundamental form, $\nabla^{\perp}$ the normal connection and $A$ the shape operator of $M$ (see [2]).

Denote by $R$ and $\widetilde{R}$ the Riemannian curvature tensors of $M$ and $N$, respectively. Then the equation of Gauss is given by

$$
\begin{aligned}
R(X, Y, Z, W) & =\widetilde{R}(X, Y, Z, W)+g(\sigma(Y, Z), \sigma(X, W)) \\
& -g(\sigma(X, Z), \sigma(Y, W)),
\end{aligned}
$$

for all vector fields $X, Y, Z, W$ tangent to $M$ (see [2]).

For any orthonormal basis $\left\{e_{1}, \ldots, e_{n}\right\}$ of the tangent space $T_{p} M$, the mean curvature vector is given by

$$
H(p)=\frac{1}{n} \sum_{i=1}^{n} \sigma\left(e_{i}, e_{i}\right),
$$

where $n=\operatorname{dim} M$.

We set $\sigma_{i j}^{r}=g\left(\sigma\left(e_{i}, e_{j}\right), e_{r}\right), i, j \in\{1, \ldots, n\}, r \in\{n+1, \ldots, m\}$, the coefficients of the second fundamental form $\sigma$ with respect to $e_{1}, \ldots, e_{n}$, 
$e_{n+1}, \ldots, e_{m}$, and

$$
\|\sigma\|^{2}=\sum_{i, j=1}^{n} g\left(\sigma\left(e_{i}, e_{j}\right), \sigma\left(e_{i}, e_{j}\right)\right) .
$$

Let $M$ be an $n$-dimensional Riemannian manifold and $\left\{e_{1}, \ldots, e_{n}\right\}$ be an orthonormal basis of $M$. For a differentiable function $f$ on $M$, the Laplacian $\Delta f$ of $f$ is denoted by $\Delta f=\sum_{j=1}^{n}\left\{\left(\nabla_{e_{j}} e_{j}\right) f-e_{j} e_{j} f\right\}$.

We will need the following Chen's Lemma for later use:

Lemma 2.1 ([3]). Let $n \geq 2$ and $a_{1}, a_{2}, \ldots, a_{n}, \lambda$ be real numbers such that

$$
\left(\sum_{i=1}^{n} a_{i}\right)^{2}=(n-1)\left(\sum_{i=1}^{n} a_{i}^{2}+\lambda\right) .
$$

Then $2 a_{1} a_{2} \geq \lambda$, with equality holding if and only if $a_{1}+a_{2}=a_{3}=\ldots=a_{n}$.

\section{Doubly warped product submanifolds}

In this section, we establish a sharp relationship between the warping functions $f_{1}$ and $f_{2}$ of a doubly warped product ${ }_{f_{2}} M_{1} \times_{f_{1}} M_{2}$ isometrically immersed in a Riemannian manifold of quasi-constant sectional curvature and the squared mean curvature $\|H\|^{2}$.

Decomposing the vector field $P$ on $M$ uniquely into its tangent and normal components $P^{\top}$ and $P^{\perp}$, respectively, we have

$$
P=P^{\top}+P^{\perp} \text {. }
$$

Now, let us begin with the following theorem:

Theorem 3.1. Let $x: f_{2} M_{1} \times_{f_{1}} M_{2} \rightarrow N$ be an isometric immersion of an n-dimensional doubly warped product ${ }_{f_{2}} M_{1} \times{ }_{f_{1}} M_{2}$ into an m-dimensional Riemannian manifold $N$ of quasi-constant curvature. Then we have:

$$
\begin{aligned}
& n_{2} \frac{\Delta_{1} f_{1}}{f_{1}}+n_{1} \frac{\Delta_{2} f_{2}}{f_{2}} \leq \frac{n^{2}}{4}\|H\|^{2}+n_{1} n_{2} a \\
& -b \sum_{1 \leq i \leq n_{1}} \sum_{n_{1}+1 \leq s \leq n}\left(T\left(e_{i}\right)^{2}+T\left(e_{s}\right)^{2}\right)+b(n-1)\left\|P^{\top}\right\|^{2},
\end{aligned}
$$

where $n_{i}=\operatorname{dim} M_{i}, n=n_{1}+n_{2}$ and $\Delta_{i}$ is the Laplacian of $M_{i}, i=1,2$. The equality case of (10) holds identically if and only if the immersion $x$ is mixed totally geodesic with $\operatorname{tr} \sigma_{1}=\operatorname{tr} \sigma_{2}$. 
Proof. Let $M={ }_{f_{2}} M_{1} \times_{f_{1}} M_{2}$ be a doubly warped product submanifold of a Riemannian manifold $N$ of quasi-constant curvature. Since $f_{2} M_{1} \times{ }_{f_{1}} M_{2}$ is a doubly warped product, we have

$$
\begin{aligned}
& \nabla_{X} Y=\nabla_{X}^{1} Y-\frac{f_{2}^{2}}{f_{1}^{2}} g_{1}(X, Y) \nabla^{2}\left(\ln f_{2}\right), \\
& \nabla_{X} Z=Z\left(\ln f_{2}\right) X+X\left(\ln f_{1}\right) Z,
\end{aligned}
$$

for any vector fields $X, Y$ on $M_{1}$ and $Z$ on $M_{2}$, where $\nabla^{1}$ and $\nabla^{2}$ are LeviCivita connections of the Riemannian metrics $g_{1}$ and $g_{2}$, respectively. Here, $\nabla^{2}\left(\ln f_{2}\right)$ denotes the gradient of $\left(\ln f_{2}\right)$ with respect to the metric $g_{2}$.

If $X$ and $Z$ are unit vector fields, it follows that the sectional curvature $K(X \wedge Z)$ of the plane section spanned by $X$ and $Z$ is given by

$$
K(X \wedge Z)=\frac{1}{f_{1}}\left\{\left(\nabla_{X}^{1} X\right) f_{1}-X^{2} f_{1}\right\}+\frac{1}{f_{2}}\left\{\left(\nabla_{Z}^{1} Z\right) f_{2}-Z^{2} f_{2}\right\} .
$$

If we choose a local orthonormal frame $\left\{e_{1}, \ldots, e_{n_{1}}, e_{n_{1}+1}, \ldots, e_{n}\right\}$ such that $e_{1}, \ldots, e_{n_{1}}$ are tangent to $M_{1}, e_{n_{1}+1}, \ldots, e_{n}$ are tangent to $M_{2}$ and $e_{n+1}$ is parallel to the mean curvature vector $H$, we obtain

$$
n_{2} \frac{\Delta f_{1}}{f_{1}}+n_{1} \frac{\Delta f_{2}}{f_{2}}=\sum_{1 \leq j \leq n_{1}<s \leq n} K\left(e_{j} \wedge e_{s}\right),
$$

for each $s \in\left\{n_{1}+1, \ldots, n\right\}$.

From the equation of Gauss, for $X=W=e_{i}$ and $Y=Z=e_{j}$ such that $i \neq j$, we have $2 \tau=n^{2}\|H\|^{2}-\|\sigma\|^{2}+2 b(n-1)\left\|P^{\top}\right\|^{2}+n(n-1) a$, where $\|\sigma\|^{2}$ is the squared norm of the second fundamental form $\sigma$ of $M$ in $N$ and $\tau$ is the scalar curvature of $M={ }_{f_{2}} M_{1} \times_{f_{1}} M_{2}$.

We set

$$
\delta=2 \tau-\frac{n^{2}}{2}\|H\|^{2}-2 b(n-1)\left\|P^{\top}\right\|^{2}-n(n-1) a .
$$

Then, we can write equation (15) as follows

$$
n^{2}\|H\|^{2}=2\left(\delta+\|\sigma\|^{2}\right) .
$$

For a chosen local orthonormal frame, the relation (16) takes the following form

$$
\left(\sum_{i=1}^{n} \sigma_{i i}^{n+1}\right)^{2}=2\left[\delta+\sum_{i=1}^{n}\left(\sigma_{i i}^{n+1}\right)^{2}+\sum_{i \neq j}\left(\sigma_{i j}^{n+1}\right)^{2}+\sum_{r=n+2}^{m} \sum_{i, j=1}^{n}\left(\sigma_{i j}^{r}\right)^{2}\right] .
$$


If we put $a_{1}=\sigma_{11}^{n+1}, a_{2}=\sum_{i=2}^{n_{1}} \sigma_{i i}^{n+1}$ and $a_{3}=\sum_{t=n_{1}+1}^{n} \sigma_{t t}^{n+1}$, then the above equation turns into

$$
\begin{aligned}
\left(\sum_{i=1}^{3} a_{i}\right)^{2}= & 2\left[\delta+\sum_{i=1}^{3} a_{i}^{2}+\sum_{1 \leq i \neq j \leq n}\left(\sigma_{i j}^{n+1}\right)^{2}+\sum_{r=n+2}^{m} \sum_{i, j=1}^{n}\left(\sigma_{i j}^{r}\right)^{2}\right. \\
& \left.-\sum_{2 \leq j \neq k \leq n_{1}} \sigma_{j j}^{n+1} \sigma_{k k}^{n+1}-\sum_{n_{1}+1 \leq s \neq t \leq n} \sigma_{s s}^{n+1} \sigma_{t t}^{n+1}\right] .
\end{aligned}
$$

Hence, $a_{1}, a_{2}$ and $a_{3}$ satisfy the Chen's Lemma (for $n=3$ ), which implies that $\left(\sum_{i=1}^{3} a_{i}\right)^{2}=2\left(\lambda+\sum_{i=1}^{3} a_{i}^{2}\right)$ with

$$
\begin{aligned}
\lambda= & \delta+\sum_{1 \leq i \neq j \leq n}\left(\sigma_{i j}^{n+1}\right)^{2}+\sum_{r=n+2}^{m} \sum_{i, j=1}^{n}\left(\sigma_{i j}^{r}\right)^{2} \\
& -\sum_{2 \leq j \neq k \leq n_{1}} \sigma_{j j}^{n+1} \sigma_{k k}^{n+1}-\sum_{n_{1}+1 \leq s \neq t \leq n} \sigma_{s s}^{n+1} \sigma_{t t}^{n+1} .
\end{aligned}
$$

Then we get $2 a_{1} a_{2} \geq \lambda$, with equality holding if and only if $a_{1}+a_{2}=a_{3}$. Equivalently, we have

$$
\begin{gathered}
\sum_{1 \leq j<k \leq n_{1}} \sigma_{j j}^{n+1} \sigma_{k k}^{n+1}+\sum_{n_{1}+1 \leq s<t \leq n} \sigma_{s s}^{n+1} \sigma_{t t}^{n+1} \\
\geq \frac{\delta}{2}+\sum_{1 \leq \alpha<\beta \leq n}\left(\sigma_{\alpha \beta}^{n+1}\right)^{2}+\frac{1}{2} \sum_{r=n+2}^{m} \sum_{\alpha, \beta=1}^{n}\left(\sigma_{\alpha \beta}^{r}\right)^{2} .
\end{gathered}
$$

Equality holds if and only if

$$
\sum_{i=1}^{n_{1}} \sigma_{i i}^{n+1}=\sum_{t=n_{1}+1}^{n} \sigma_{t t}^{n+1}
$$

By making use of the Gauss equation again, we have

$$
n_{2} \frac{\Delta_{1} f_{1}}{f_{1}}+n_{1} \frac{\Delta_{2} f_{2}}{f_{2}}=\tau-\sum_{1 \leq j<k \leq n_{1}} K\left(e_{j} \wedge e_{k}\right)-\sum_{n_{1}+1 \leq s<t \leq n} K\left(e_{s} \wedge e_{t}\right)
$$




$$
\begin{aligned}
& =\tau-\frac{n_{1}\left(n_{1}-1\right)}{2} a-\sum_{r=n+1}^{m} \sum_{1 \leq j<k \leq n_{1}}\left[\sigma_{j j}^{r} \sigma_{k k}^{r}-\left(\sigma_{j k}^{r}\right)^{2}\right] \\
(19) & -b \sum_{1 \leq j<k \leq n}\left(T\left(e_{k}\right)^{2}+T\left(e_{j}\right)^{2}\right)-\frac{n_{2}\left(n_{2}-1\right)}{2} a \\
& -\sum_{r=n+1}^{m} \sum_{n_{1}+1 \leq s<t \leq n_{1}}\left[\sigma_{s s}^{r} \sigma_{t t}^{r}-\left(\sigma_{s t}^{r}\right)^{2}\right]-b \sum_{n_{1}+1 \leq s<t \leq n}\left(T\left(e_{s}\right)^{2}+T\left(e_{t}\right)^{2}\right) .
\end{aligned}
$$

In view of the equations (14), (17) and (19) we obtain

$$
\begin{aligned}
& n_{2} \frac{\Delta_{1} f_{1}}{f_{1}}+n_{1} \frac{\Delta_{2} f_{2}}{f_{2}} \leq \tau-\frac{n(n-1)}{2} a+n_{1} n_{2} a-\frac{\delta}{2} \\
& -\frac{1}{2} \sum_{r=n+2}^{m} \sum_{\alpha, \beta=1}^{n}\left(\sigma_{\alpha \beta}^{r}\right)^{2}+\sum_{r=n+2}^{m} \sum_{1 \leq j<k \leq n_{1}}\left[\left(\sigma_{j k}^{r}\right)^{2}-\sigma_{j j}^{r} \sigma_{k k}^{r}\right] \\
& +\sum_{r=n+2}^{m} \sum_{n_{1}+1 \leq s<t \leq n_{1}}\left[\left(\sigma_{s t}^{r}\right)^{2}-\sigma_{s s}^{r} \sigma_{t t}^{r}\right]-b \sum_{1 \leq j<k \leq n}\left(T\left(e_{k}\right)^{2}+T\left(e_{j}\right)^{2}\right) \\
& -\quad b \sum_{n_{1}+1 \leq s<t \leq n}\left(T\left(e_{s}\right)^{2}+T\left(e_{t}\right)^{2}\right) \\
& \text { (20) }=\tau-\frac{n(n-1)}{2} a+n_{1} n_{2} a-\frac{\delta}{2}-\sum_{r=n+1}^{m} \sum_{j=1}^{n_{1}} \sum_{t=n_{1}+1}^{n}\left(\sigma_{j t}^{r}\right)^{2} \\
& -\frac{1}{2} \sum_{r=n+2}^{m}\left(\sum_{j=1}^{n_{1}} \sigma_{j j}^{r}\right)^{2}-\frac{1}{2} \sum_{r=n+2}^{m}\left(\sum_{t=n_{1}+1}^{n} \sigma_{t t}^{r}\right)^{2} \\
& -b \sum_{1 \leq j<k \leq n}\left(T\left(e_{k}\right)^{2}+T\left(e_{j}\right)^{2}\right)-b \sum_{n_{1}+1 \leq s<t \leq n}\left(T\left(e_{s}\right)^{2}+T\left(e_{t}\right)^{2}\right) \\
& \leq \tau-\frac{n(n-1)}{2} a+n_{1} n_{2} a-\frac{\delta}{2} \\
& -b \sum_{1 \leq j<k \leq n}\left(T\left(e_{k}\right)^{2}+T\left(e_{j}\right)^{2}\right)-b \sum_{n_{1}+1 \leq s<t \leq n}\left(T\left(e_{s}\right)^{2}+T\left(e_{t}\right)^{2}\right)
\end{aligned}
$$

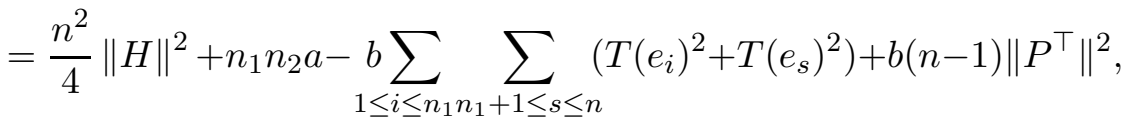

which gives us (10). 
By using of (18) and (20), it can be easily seen that the equality sign of (10) holds if and only if

$$
\sigma_{j t}^{r}=0, \quad n+1 \leq r \leq m
$$

and

$$
\sum_{i=1}^{n_{1}} \sigma_{i i}^{r}=\sum_{t=n_{1}+1}^{n} \sigma_{t t}^{r}=0
$$

for $1 \leq j \leq n_{1}, n_{1}+1 \leq t \leq n$ and $n+2 \leq r \leq m$. The equation (21) means that the second fundamental form $\sigma$ of $f_{2} M_{1} \times_{f_{1}} M_{2}$ in $N$ is as follows $\sigma\left(D_{1}, D_{2}\right)=\{0\}$. Hence, the immersion $x$ is mixed totally geodesic. From (18) and (22), we also get $\sum_{j=1}^{n_{1}} \sigma\left(e_{j}, e_{j}\right)=\sum_{s=n_{1}+1}^{n} \sigma\left(e_{s}, e_{s}\right)$, which implies that $\operatorname{tr} \sigma_{1}=\operatorname{tr} \sigma_{2}$.

Conversely, assume that $N$ is an $m$-dimensional Riemannian manifold of quasi-constant curvature and the immersion $x$ is mixed totally geodesic with $\operatorname{tr} \sigma_{1}=\operatorname{tr} \sigma_{2}$. Then, inequalities (17) and (20) reduce to equalities. Hence, we obtain the equality sign of (10) and finish the proof of the theorem.

As a consequence of Theorem 3.1 we can give the following corollary:

Corollary 3.2. Let $x: f_{2} M_{1} \times_{f_{1}} M_{2} \rightarrow N$ be an isometric immersion of an $n$-dimensional doubly warped product ${ }_{f_{2}} M_{1} \times_{f_{1}} M_{2}$ into an $m$-dimensional Riemannian manifold $N$ of quasi-constant curvature. If the vector field $P$ is tangent to $M$, then we have:

$$
\begin{aligned}
n_{2} \frac{\Delta_{1} f_{1}}{f_{1}}+n_{1} \frac{\Delta_{2} f_{2}}{f_{2}} \leq & \frac{n^{2}}{4}\|H\|^{2}+n_{1} n_{2} a+b(n-1) \\
& -b \sum_{1 \leq i \leq n_{1}} \sum_{n_{1}+1 \leq s \leq n}\left(T\left(e_{i}\right)^{2}+T\left(e_{s}\right)^{2}\right) .
\end{aligned}
$$

If the vector field $P$ is normal to $M$, then we have:

$$
n_{2} \frac{\Delta_{1} f_{1}}{f_{1}}+n_{1} \frac{\Delta_{2} f_{2}}{f_{2}} \leq \frac{n^{2}}{4}\|H\|^{2}+n_{1} n_{2} a
$$

The equality case of (23) and (24) holds identically if and only if the immersion $x$ is mixed totally geodesic with $\operatorname{tr} \sigma_{1}=\operatorname{tr} \sigma_{2}$. 


\section{REFERENCES}

1. Chaki, M.C.; Maity, R.K. - On quasi Einstein manifolds, Publ. Math. Debrecen, 57 (2000), 297-306.

2. Chen, B.-Y. - Geometry of Submanifolds, Pure and Applied Mathematics, No. 22, Marcel Dekker, Inc., New York, 1973.

3. Chen, B.-Y. - Some pinching and classification theorems for minimal submanifolds, Arch. Math. (Basel), 60 (1993), 568-578.

4. Chen, B.-Y. - Some new obstructions to minimal and Lagrangian isometric immersions, Japan. J. Math. (N.S.), 26 (2000), 105-127.

5. Chen, B.-Y. - On isometric minimal immersions from warped products into real space forms, Proc. Edinb. Math. Soc., 45 (2002), 579-587.

6. Chen, B.-Y.; Yano, K. - Hypersurfaces of a conformally flat space, Tensor (N.S.), 26 (1972), 318-322.

7. Olteanu, A. - A general inequality for doubly warped product submanifolds, Math. J. Okayama Univ., 52 (2010), 133-142.

8. ÖzGür, C.; Murathan, C. - Warped product submanifolds of a Riemannian manifold of quasi-constant curvature, preprint.

9. Sular, S.; ÖzGür, C. - Doubly warped product submanifolds of $(\kappa, \mu)$-contact metric manifolds, Ann. Polon. Math., 100 (2011), 223-236.

10. UnAL, B. - Doubly warped products, Thesis (Ph.D.), University of Missouri, Columbia, 2000.

Received: 15.III.2012

Revised: 17.V.2012

Accepted: 25.V.2012
Department of Mathematics, Balıkesir University, 10145, Çă̆ıs, Balıkesir, TURKEY csibel@balikesir.edu.tr 